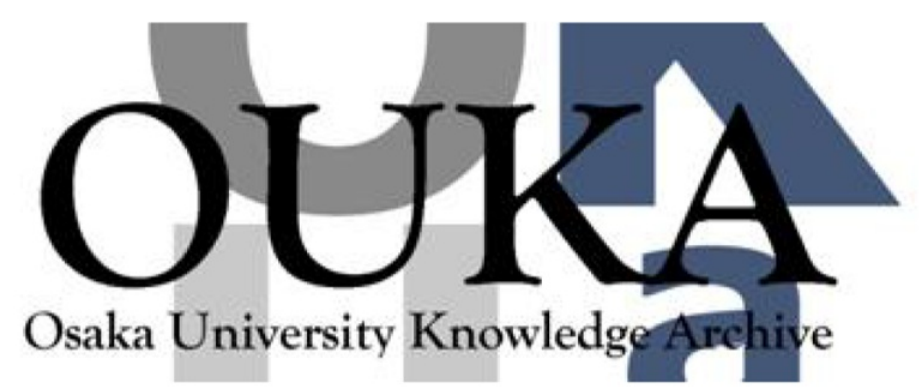

\begin{tabular}{|c|l|}
\hline Title & $\begin{array}{l}\text { Optical observation of subbands in amorphous } \\
\text { silicon ultrathin single layers }\end{array}$ \\
\hline Author(s) & Hattori, K.; Mori, T.; Okamoto, H. et al. \\
\hline Citation & Applied Physics Letters. 53(22) p. 2170-p. 2172 \\
\hline Issue Date & $1988-11-28$ \\
\hline oaire:version VoR \\
\hline URL & https://hdl. handle. net/11094/3084 \\
\hline rights & \\
\hline Note & \\
\hline
\end{tabular}

Osaka University Knowledge Archive : OUKA

https://ir. Library. osaka-u. ac. jp/

Osaka University 


\title{
Optical observation of subbands in amorphous silicon uitrathin single layers
}

\author{
K. Hattori, T. Mori, H. Okamoto, and Y. Hamakawa \\ Faculty of Engineering Science, Osaka University, Toyonaka, Osaka 560, Japan
}

(Reccived 13 June 1988; accepted for publication 22 September 1988)

Wavelength-differential absorption spectroscopy has been applied to amorphous silicon ultrathin single layers. The derivative spectra clearly show a step-like behavior for the layer of the thickness below $40 \AA$, indicating a splitting of the valence and conduction bands into a series of the subbands. Analysis of the observed energy shifts of subband transitions in terms of a one-dimensional quantum well model leads to the conclusion that the electron effective mass is less than $0.48 m_{0}$, where $m_{0}$ denotes free electron mass.

The most profound consequence of quantum confinement of carriers in uitrathin semiconductor layers is the appearance of two-dimensional subbands, which manifest themselves in peculiar electrical and optical effects. For hydrogenated amorphous silicon ( $a-\mathrm{Si}: \mathrm{H}$ ), the presence of subbands has been directly identified in the resonance tunneling characteristics of $a-\mathrm{SiN}: \mathrm{H} / a-\mathrm{Si}: \mathrm{H} / a-\mathrm{SiN}: \mathrm{H}$ double-barrier diodes ${ }^{\mathrm{I}}$ as well as in the differential optical absorption spectra of $\alpha$-Si:H/a-SiC:H multilayered structures. ${ }^{2}$ Preparation technologies are well established for these multiple layered structures to control the transition of material compositions with an accuracy of a few atom spacings. However, if the simple square quantum well model is to be applied for the analysis of the observed subband energies, the interface abruptness of this extert may be still insufficient since the quantization efects appear to become remarkable oniy when the sublayer thickness is reduced to less than several tens angstroms.

As contrasted with the multiple layered structures, $a$-Si:H single layers deposited on $\mathrm{SiO}_{2}$ glass offer an extended possibility for investigating the quantum confinement effect in a nearly ideal deep square potential well formed by abrupt vacuum/ $a$-Si:H and $a$-Si: $\mathrm{H} / \mathrm{SiO}_{2}$ glass interfaces. ${ }^{3}$ In this work, we have carried out such study, using the wavelength-differential absorption (WDA) technique. The measured WDA spectrum exhibits a step-like behavior easily attributed to the subband transitions wher the layer thickness decreased to less than $40 \AA$. The experimental data for energy shifts of the subband transitions are discussed in accordance with a one-dimensional quantum well model.

In the experiment, $a$-Si:H layers varying in thickness from 20 to $60 \mathrm{~A}$ were prepared by rf plasma chemical vapor deposition with a $1: 9 \mathrm{SiH}_{4}-\mathrm{H}_{2}$ gas mixture at a substrate temperature $300^{\circ} \mathrm{C}$. One set of the samples was grown directly on a syrthesized $\mathrm{SiO}_{2}$ glass $\left(\mathrm{G}-\mathrm{SiO}_{2}: \mathrm{K}\right.$. $\left.\mathrm{K}\right)$ substrate, while as a reference sample the $\alpha$-Si:H layer was sandwiched with $a$-SiC: $Y$ layers of $50 \AA$ thickness. The carbide barrier layers were grown from using a $1: 11$ :108 $\mathrm{SiH}_{4}-\mathrm{CH}_{4}-\mathrm{H}_{2}$ gas mixture. The $a-S i: H$ and $a-S i C$ :H layers were formed in separate chambers with an interruption on the piasma. The layer thicknesses were determined by the deposition time and the deposirion rates for the thick fims. The optical band gaps were $1.75 \mathrm{eV}$ for $a-S i: H$ and $2.80 \mathrm{eV}$ for $a-S i C: H$, respectively. After cooling to room temperature, the samples were mounted in an evacuated optical cryostat. In the WDA measurement, focused broadband light with an intensity 5
$\mathrm{mW} / \mathrm{cm}^{2}$ was directed onto the sample, and the transmitted light was dispersed and detected using a monochromator and a photomutiplier. From the transmittance $T$ and its wavelength $(\lambda)$ derivative $d T / d \lambda$, the WDA signal was obtained in the form of $(d T / d \lambda) / T$ in the photon-energy range $1.7-2.4 \mathrm{eV}$. The measurement was performed at room temperature $(293 \mathrm{~K})$ in the present work.

We will here briefy discuss the WDA spectrum expected for the quantum regime. Optical transitions between subband states should obey an approximate conservation rule for the confined-state quantum number $n(\Delta n=0)$. Therefore, the absorption coefficient $\alpha$ in the amorphous semiconductor quantum well is approximately represented by

$$
\hbar \omega c \propto \sum_{n}\left(\hbar \omega-E_{n}\right) U\left(\hbar \omega-E_{n}^{2}\right), \quad n=1,2,3, \ldots,
$$

within the context of a model assuming a relaxed selection rule for wave vector $\mathrm{k}$ in the direction parallel to the well plane as well as a constant momentum matrix element for the transitions. ${ }^{2}$ Here, $\hbar \omega$ denotes photon energy ${ }_{2} E_{n}$ energy separation between the $n$th subband edges in the conduction and valerice bands, and $U(E)$ a step function. Equation ( 1 ) indicates that the quantization effect does not produce a staircase structure as does in quantum wells based upon crystalline direct gap semiconductors, instead only changes in the slope are induced at each transition energy $E_{n}$. This weakened feature associated with the subband transitions appears to be greatly enhanced in the derivatives. The wavelength derivative of the absorption spectrum is expressed by

$$
\frac{d \alpha}{d \lambda} \propto-(\hbar \omega)^{2} \frac{d \alpha}{d(\hbar \omega)} \propto-\sum_{n} E_{n} U\left(\hbar \omega-E_{n}\right),
$$

suggesting that the spectrum exhibits a staircase form with steps at photon energies $n=E_{n}$. On the other hand, the expression for a three-dimensional parabolic-band transition in the unquantization regime is given using a Tauc cptical band gap $E_{0}$ by

$$
\frac{d \alpha}{d \lambda} \alpha-\left[\left(h_{\omega} \omega\right)^{2}-E_{0}^{2}\right\rceil U\left(\hbar \omega-E_{0}\right),
$$

when the compietely relaxed $k$-selection rule in three dimensions is assumed. In this case, the spectrum shows only a smooth increase with photon energy. The WDA spectrum $(d T / d \lambda) / T$ is directly related to the derivative of absorption coefficient $d \alpha / d \lambda$ so far as the refractive index is a siowly 
varying function of wavelength as compared with the absorption coefficient.

Figure 1 presents the WDA spectra measured on the $a$-Si:H single layers of thicknesses $L=20,25,40$, and $60 \AA$, as a function of photon energy $h \omega$. A structureless spectrum is observed for $L=60 \AA$, which approximately follows relation (3) for the unquantization regime. The spectral shapes for $L \leqslant 40 \AA$ are entirely different from that for $L=60 \AA$. It is clearly found that the spectra have step-like structures ascribed to subband transitions. The successful detection of well-resolved step edges even at room temperature is likely to suggest that the $a$-Si:H single layer is of uniform thickness and of nearly ideal electronic property of the untreated surface and interface, at which dangling bonds would be terminated by hydrogen atoms. On the other hand, gradual increases found at the higher photon energy region in the WDA spectra are considered to be caused mainly by the effect of the wavelength-dependent refractive index. The energy positions of step edges, which are to be identified as the subband transition energies $E_{n}$, are indicated by arrows in the figure. The observed blue shift of the first absorption edge $E_{1}$ with decreasing layer thickness is consistent with that of photoluminescence peak reported on a similar sample configuration. ${ }^{4}$ A nearly identical energy position for $(n=l, L=20 \AA)$ and $(n=2, L=40 \AA)$ matches with the implication from a simple model for a suffeiently deep potential well, in which $E_{n}$ is approximately determined by the factor $n / L$.

The energy levels in a quantum well are controlled by changing the well width and also the potential barriers at both sides. As already pointed out above, the quantized ener-

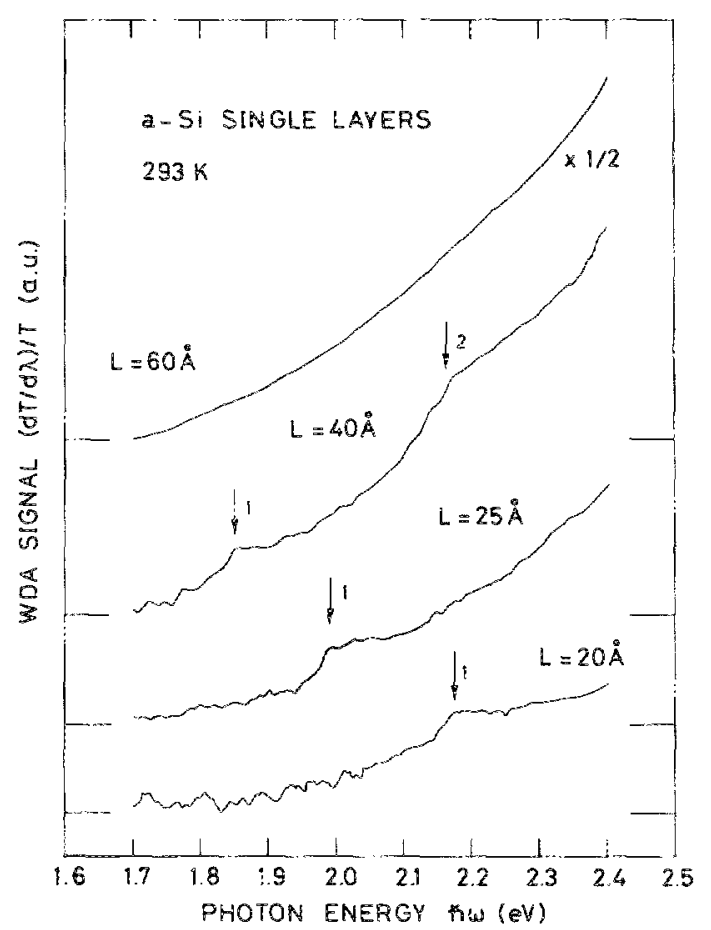

FIG. 1. WDA spectra $(d T / d \lambda) / T$ measured on $a$-Si:H single layers with thicknesses $L=20,25,40$, and $60 \AA$ at temperature $293 \mathrm{~K}$. The arrows indicate the energy positions of step edges.

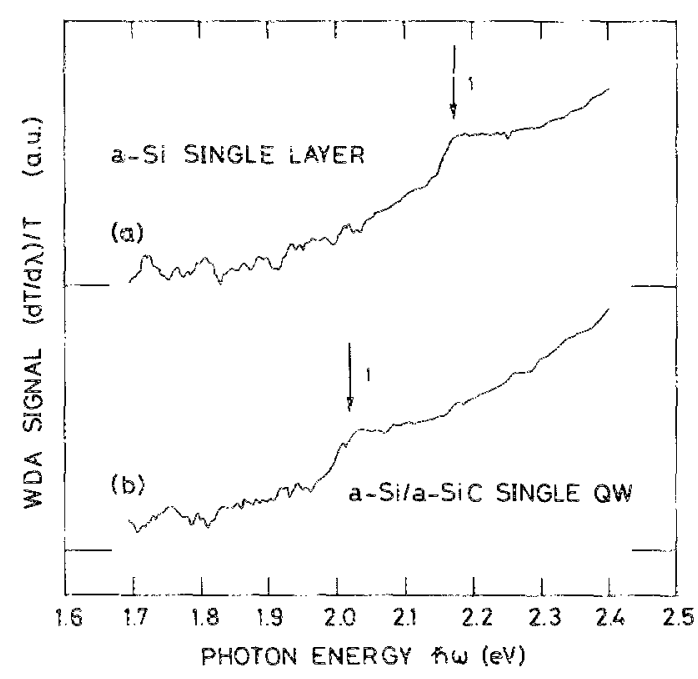

FIG. 2. WDA spectra measured on (a) $a$-Si:H $(20 \AA)$ single layer and (b) $a-\operatorname{SiC}: \mathrm{H}(50 \AA) / a-S i: H(20 \AA) / a-5 i C: H(50 \AA)$ sandwich structure at temperature $293 \mathrm{~K}$. The arrows indicate the energy positions of the first step edge.

gy level formed in the single layer shows a systematic shift by varying the layer thickness.

We next turn our focus to the difference in energy levels of quancum well structures constructed with difierent barrier materials. Figure 2 shows the WDA spectra measured on (a) the $a-\mathrm{Si}: \mathrm{H}$ single layer and (b) the $a-\mathrm{SiC}: \mathrm{H} /$ $a-\$ i: H / a-S i C: H$ sandwich structure (single quantum well), where both samples have the same $a$-Si.H well width of $20 \AA$. The subband transition energy $(n=1)$ for the $a-S i C: H$ barrier sample is found to be smaller than that for the vacum and the $a-\mathrm{SiO}_{2}: \mathrm{H}$ barrier. The result is interpreted as a natural consequence of the smaller potential barriers formed by the $a-\mathrm{Si}: \mathrm{H} / \mathrm{a}-\mathrm{SiC}: \mathrm{H}$ helerojunction interface ${ }^{2}$ relative to those built up by the vacuum/a-Si:H and $a-\mathrm{Si}: \mathrm{H} / a-\mathrm{SiO}_{2}: \mathrm{H}$ interfaces. $^{3}$

In Fig. 3 the energy shifts of subband transitions, $E_{n}$

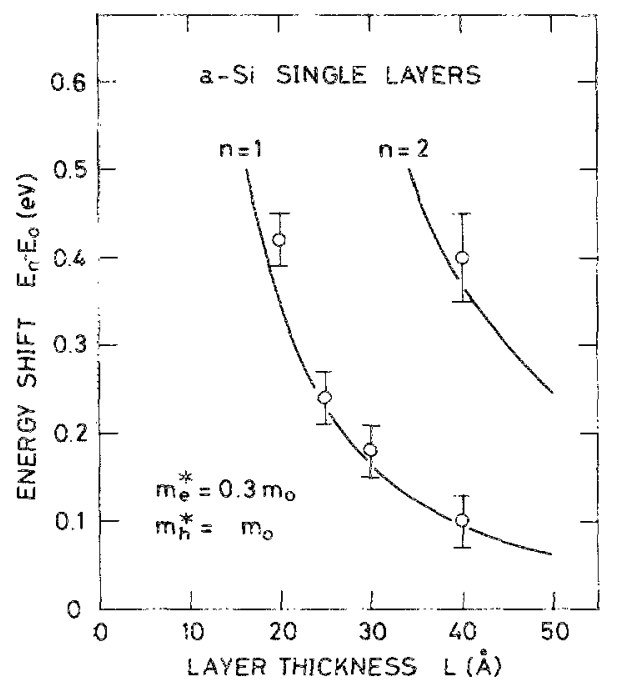

FIG. 3. Energy shifts of subband transitims $E_{n}-E_{0}(n=1$ and 2$)$ measured on $a-\mathrm{Si} H \mathrm{H}$ single layers as function of thickness $L$. Circles are experimental data, and solid lines the theoretical plots calculated from a one-cimensional quantum well model. 
$-E_{0}(n=1$ and 2$)$, observed for the $a$-Si:H single layers, are summarized as a function of the layer thickness $L$. The cnergy shift is easily calculated from the one-dimensional quantum well model, assuming that the band discontinuities at the $a-\mathrm{S}: \mathrm{H} / \mathrm{a}-\mathrm{SiO}_{2}: \mathrm{H}$ interface are $4 \mathrm{eV}$ for the valence band ${ }^{3}$ and $3.25 \mathrm{eV}$ for the conduction band. The result of the calculation is plotted by solid lines in the figure, where the elccron $\left(m_{*}^{*}\right)$ and hole $\left(m_{h}^{*}\right)$ effective masses are chosen as $0.3 m_{0}$ and $m_{0}$, respectively, in boti $a-5 i: H$ and $a-\$_{2} O_{2}: H$ regions. As found in the figure, the theoretical curves yield reasonabie fits to the experimental data. The identical values of effective masses are deduced from our recent work on $a-$ Si:H/ $a-$ SiC:H multiple quantum well structures. ${ }^{2}$

The estimation of the effective masses described above, however, may involve some uncertainty due to the choice of parameters used, such as barrier heights. To remove the ambiguity, here we will make another simpler approach for the estimation. The transition energy $E_{n}$ in an infinitely deep square well is expressed by a well-known relation: $E_{n}=E_{0}$ $+\left(\hbar^{2} / 2 m m_{r}^{*}\right)(n \pi / L)^{2}$, where $m_{r}^{*}$ denotes the reduced effective mass of electrons and holes. The experimental data are found to approximately satisfy this relation, providing the upper limit of the reduced mass is in the $a-\mathrm{Si}: \mathrm{H}$ layer. The value is deduced to be $0.24 m_{0}$. Assuming the electron effective mass $m_{c}^{*}$ to be smaller than the hole effective mass $m_{h}^{*}$ leacs to a conclusion that the $m_{*}^{*}$ must be less than $0.48 m_{0}$. The assumption for the effective masses $m_{e}^{*}<m_{h}^{*}$ does not contradict with the fact that the density of states at the bottom of the conduction band is lower than that at the top of the valence band, ${ }^{5}$ and the extended-state electron mobility is larger than that of holes. ${ }^{6}$ In contrast with the present estimation, a larger electron mass $\left(m_{c}^{*}=0.6 m_{0}\right)$ is suggested from the analysis of the resonant tunneling phenomenon in $a-\mathrm{SiN}: \mathrm{H} / a-\mathrm{Si}: \mathrm{H} / a-\mathrm{SiN}: \mathrm{H}$ double-barrier structures. ${ }^{3}$ This disagreement may imply that the electron mass in the $a$-Sin:H barrier layer takes a larger value than that in the a-Si.H layer.

In summary, WDA spectra of the $a-S i: B$ single layers have been investigated with varying layer thicknesses. When the layer thickness decreases to less than $40 \AA$, the spectrum appears to exhibit a step-ike behavior, indicating that the valence and conduction bands split into two-dimensional subbands. The experimental data for the energy shift of subband transitions are in quantitative agreement with those expected from the one-dimensional quantum well model, suggesting that the electron effective mass is less than $0.48 m_{0}$, or specifcally $0.3 m_{0}$. Since the concept of effective mass is not established in amorphous semiconductors, any firm conchision cannot be drawn from the deduced quantity. It is, however, of interest to note that the directional averaged effective mass takes a value close to $0.3 m_{0}$ in crystalline Si, SiC ( $3 \mathrm{C}$ and $6 \mathrm{H}$ ), $\mathrm{C}$ (diamond), and $\mathrm{SiO}_{2}$. The impication from this apparent coincidence might be important for understanding the electronic structure of $a-\$ i$. H and its alloy materials.

'S. Miyazaki, Y. Ihara, and M. Hirose, Phys. Rev. Lett. 59, 125 (1987).

${ }^{2} \mathrm{~K}$. Hattori, T. Mori, H. Okamoto, and Y. Lamakawa, Phys. Rev. Lett. 60 , 825 (1988).

'L. Yang, B. Abeles, W. Eberhardt, and H. Stasiewski, Fhys. Rev. B 35, 9395 (1987).

4B. A. Wilson, C. M. Taylor, and I. B. Harbison, Phys. Rev. B 34, 4429 (1986).

${ }^{5}$ D. Zdetsis, L. Economu, D. A. Papaconstantopoulus, and N. Flytzanis, Phys. Rev. B 31, 2410 (1985).

'T. Tiedje, J. M. Cebulka, D. L. Morel, and B. Abeles, Phys. Kev. Lett. 46, $1425(1981)$. 